\title{
A room temperature cured low dielectric hyperbranched epoxy adhesive with high mechanical strength
}

\author{
BIBEKANANDA DE and NIRANJAN KARAK* \\ Advanced Polymer and Nanomaterial Laboratory, Department of Chemical Sciences, Tezpur University, \\ Napaam 784 028, India \\ e-mail: karakniranjan@yahoo.com
}

MS received 25 July 2013; revised 1 November 2013; accepted 26 November 2013

\begin{abstract}
A low dielectric constant hyperbranched epoxy thermoset with excellent adhesive and mechanical strength is the demand for advanced electronics and engineering applications. The present investigation provided a room temperature, curable hyperbranched epoxy, obtained by an $\mathrm{A}_{2}+\mathrm{B}_{3}$ polycondensation reaction. The synthesized hyperbranched epoxy was cured by a combined hardener system consisting of a commercial poly(amido-amine) and a first generation aliphatic poly(amido-amine) dendrimer (PAD) prepared by Michael addition reaction of methyl acrylate and aliphatic amines. The thermoset exhibited high mechanical strength, excellent adhesive strength, low dielectric constant, good thermal stability and excellent weather resistance along with very good moisture resistance. The results showed the influence of the amount of PAD on the performance of the thermoset. Thus, the study revealed that the combined poly(amido-amine) cured hyperbranched epoxy has high potential in advanced electrical packaging and microelectronic devices.
\end{abstract}

Keywords. Hyperbranched epoxy; poly(amido-amine) dendrimer; curing agent; low dielectric constant; high performance.

\section{Introduction}

Epoxy resins have a broad range of applications including binder for coatings, high performance composites, adhesives, insulating materials, encapsulating and packaging materials for electronic devices, flooring materials, water proofing materials, sealer, and so on. This is because of their favourable mechanical strength, good weather and chemical resistance, low shrinkage and high adhesive strength. ${ }^{1-4}$ Epoxy resins have also a lot of scientific and technical interests because of their interesting and wide possibilities of crosslinking reactions. To achieve well-balanced ultimate properties, epoxy resins have to be transformed to threedimensional network structures in the presence of suitable curing agents under optimal curing and processing conditions. Among the different types of curing agent, amines based are of prime significance for their tailoring curing conditions and ultimate performance of the thermosets. However, to overcome the drawbacks of aliphatic and aromatic primary amines, aliphatic poly(amido-amine), a resinous hardener system is largely used as the suitable curing agent in various commercial applications of epoxy resins. It cures epoxy resins with relatively low isotherm, offers long

\footnotetext{
*For correspondence
}

pot life and produces a highly plasticized thermostable finished product because of the presence of long hydrocarbon chain in its structure. ${ }^{2}$ In the present study, a liquid poly(amido-amine) dendrimer with low volatility was used to cure epoxy resin at room temperature. However, the use of only such agent results highly crosslinked brittle product. This is due to the dendritic architecture with large number of freely exposed end amine functionality which can be rapidly reacted with epoxy groups of the epoxy resin. Thus, a combined system of dendrimer and linear poly(amidoamine) was used for the curing of a hyperbranched epoxy resin at room temperature to obtain high performance thermoset for advanced engineering applications. Here the used poly(amido-amine) dendrimer was prepared by Michael addition reaction as reported by Tomalia et al. ${ }^{5}$ Though curing kinetics of bisphenol-A based epoxy resin with 1 st to 4 th generations of dendritic poly(amido-amine) with different amines were studied by DSC, ${ }^{6}$ but performance of the thermosets was not reported. Thus a detail and systematic study on curing at room temperature of a hyperbranched epoxy with a 1st generation amido-amine in combination with the linear poly(amido-amine) and the performance of the thermoset were investigated in the present study.

In the present study, the hyperbranched epoxy resin and poly(amido-amine) dendrimer were used to enjoy 
the advantages of both the structures over the conventional linear ones. The easy synthetic accessibility, low viscosity, high solubility and large number of end functional groups are a few to mention of those advantages., ${ }^{2,7-11}$ Hyperbranched epoxy resins have been extensively investigated by various synthetic approaches. ${ }^{12-15}$ However, the reported hyperbranched epoxy resins were either solid or liquid with high viscosity and thus were diluted with organic solvents during their processing and hence caused detrimental effect to the environment (high VOC). In most of the cases the mechanical strength was also found to be low and thus they were mainly used as toughening agent for diglycidyl ether of bisphenol-A based epoxy. ${ }^{12,13}$ The hyperbranched epoxy thermosets with desired performance, therefore, are limited and hence needs special attention for their development.

Further, along with room temperature curability and other desired properties, the epoxy thermosets with low dielectric constant are the demand of the days. Low dielectric constant containing epoxy thermosets are preferred for several electrical applications, especially printed circuit boards, semiconductor devices, microelectronic devices, electronic packages, etc. ${ }^{16-18}$ The use of low dielectric epoxy is advantageous, in part, because they adhere well to the surface of metals and other materials over the conventional dielectric materials. However, most of the reported epoxy thermosets possess relatively high dielectric constant in the range of 4-5 without addition of any filler, where desired dielectric constant is $\sim 3$. Thus, design of an epoxy thermoset with desired low dielectric constant along with high mechanical strength, excellent adhesive strength and good thermal stability has a great demand in recent time.

In the present work, a hyperbranched epoxy was cured with a combined hardener of poly(amidoamine) dendrimer and linear poly(amido-amine) to obtain a thermoset with high tensile strength, adequate toughness, good thermal stability, excellent adhesive strength, low dielectric constant and good chemical resistance.

\section{Experimental}

\subsection{Materials}

Triethanol amine (Merck, India) was used after vacuum drying. Bisphenol-A, epichlorohydrin and methyl acrylate were purchased from G S Chemical, Mumbai. Bisphenol-A was re-crystallized from toluene before use. Butane 1, 4-diamine (Sigma-Aldrich, Germany), ethylene diamine (Sigma-Aldrich, Germany), sodium hydroxide (Rankem, New Delhi) and poly(amidoamine) hardener (HY840, Ciba Geigy, Mumbai, amine value 5 eq. $/ \mathrm{kg}$, viscosity 14 Pas) were used as received without any further purification. All other reagents used in the present investigation were of reagent grade.

\subsection{Preparation of first generation poly(amido-amine) dendrimer}

The first generation of poly(amido-amine) dendrimer (PAD) was prepared by Michael addition reaction of amine with acrylate following the literature procedure. ${ }^{5}$ Briefly, in a three neck $100 \mathrm{~mL}$ round bottom flask equipped with a water condenser, a thermometer and a dropping funnel, $10 \mathrm{~mL}$ methanolic solution of $2 \mathrm{~g}$ (0.0226 mol) 1,4-butane diamine was taken. In this solution, $15.62 \mathrm{~g}(0.18 \mathrm{~mol})$ of methyl acrylate was added drop-wise at room temperature under constant stirring. The reaction was carried out for $72 \mathrm{~h}$ at room temperature. The methanol and the excess methyl acrylate were removed under vacuum at room temperature. In the second step, $20 \mathrm{~mL}$ methanolic solution of $13.63 \mathrm{~g}(0.2268 \mathrm{~mol})$ ethylene diamine was added dropwise into the resulted product of the first step at room temperature under constant stirring. The reaction was again carried out for $72 \mathrm{~h}$. Methanol and the excess amine were removed similarly under vacuum at room temperature. The final product was soluble in water, DMSO, ethanol and methanol. So the product was purified by dissolved in water and precipitated in THF. The final yield of the dried purified product was $96 \%$ with very low viscosity 0.98 Pas.

\subsection{Preparation of the hyperbranched epoxy}

The hyperbranched epoxy was prepared by $\mathrm{A}_{2}+$ $\mathrm{B}_{3}$ polycondensation reaction of bisphenol- $\mathrm{A}$ and epichlorohydrine with triethanol amine (20 wt\% with respect to bisphenol-A) as reported earlier. ${ }^{19}$ The reaction was carried out at $110^{\circ} \mathrm{C}$ for $4 \mathrm{~h}$ using aqueous $\mathrm{NaOH}$ as the catalyst. The general structure of the hyperbranched epoxy (figure S1) is given in electronic supplementary information section. The degree of branching, epoxy equivalent and hydroxyl value of the hyperbranched epoxy was $0.79,358 \mathrm{~g} / \mathrm{eq}$. and $102 \mathrm{mg} \mathrm{KOH} / \mathrm{g}$, respectively.

\subsection{Curing study}

The hyperbranched epoxy was cured with the combined hardener system consisting of $20 \mathrm{wt} \%$ of poly(amido-amine) and 5, 10 and $15 \mathrm{wt} \%$ of PAD, 
separately. The thermosets are coded as PAD5, PAD10 and PAD15 as shown in the table 1. The hyperbranched epoxy cured with only PAD (10 wt $\%)$ coded as OPAD. The amount of hardener was calculated from the epoxy equivalent and shown in electronic supplementary information. An amount of the hyperbranched epoxy resin was homogeneously mixed with the desired amounts of both the hardeners at room temperature. This mixture was coated on mild glass plates $(75 \mathrm{~mm} \times 25 \mathrm{~mm} \times 1.3 \mathrm{~mm})$ for scratch hardness and tensile test, and on steel plates $(150 \mathrm{~mm} \times 50 \mathrm{~mm} \times 1.6 \mathrm{~mm})$ for impact resistance test. Then the plates were cured at room temperature for $24 \mathrm{~h}$ before measuring the properties. However, only linear poly(amido-amine) hardened coated plates were cured at $100^{\circ} \mathrm{C}$ for $50 \mathrm{~min}$ followed by post cured at $130^{\circ} \mathrm{C}$ for $20 \mathrm{~min}$ and coded as PAD0.

\subsection{Characterizations}

FTIR spectrum of the PAD was recorded with a Nicolet FTIR spectrophotometer (Impact-410, Madison, USA) using $\mathrm{KBr}$ pellet and NMR spectrum was recorded with a $400 \mathrm{MHz}$, JEOL FTNMR spectrometer by using $\mathrm{d}_{6}$ DMSO as the solvent and TMS as the internal standard. The physical properties and swelling value of the hardener were measured by the standard test methods. ${ }^{20}$ The mechanical properties such as scratch hardness was carried out by scratch hardness tester (Sheen Instrument Ltd., UK) and impact strength by Impact tester (S C Dey Co., Kolkata) as per the standard falling weight (ball) method (standard ASTM D 1709). In this test a weight of $850 \mathrm{~g}$ was allowed to fall on the film coated on a mild steel plate from minimum to maximum falling heights. The maximum height was taken as the impact resistance up to which the film was not damaged. The tensile and adhesion test of the cured films were carried out by Universal Testing Machine (UTM, WDW10, Jinan, China) with a
$10 \mathrm{kN}$ load cell and crosshead speed of $50 \mathrm{~mm} / \mathrm{min}$. The adhesion test was carried out on two interfaces of adherents, viz., metal-metal (M-M) and wood-wood (W-W) by lap-shear test where the area of the overlapping zone was $25 \times 25 \mathrm{~mm}^{2}$ and thickness of the adhesive layer was $0.02-0.03 \mathrm{~mm}$. The lap-shear tensile strength $(\mathrm{MPa})$ or adhesive strength was calculated as maximum load per unit bond area, obtained directly from the UTM. The thermal stability of the samples were studied by thermogravimetric analysis (TGA) in Shimazdu TG 50 using nitrogen flow rate of $30 \mathrm{~mL} / \mathrm{min}$ at the heat rate of $10^{\circ} \mathrm{C} / \mathrm{min}$ from room temperature to $700^{\circ} \mathrm{C}$. The dielectric property of the thermoset was measured by LCR Hitester instrument (Hioki 3532-50) up to a frequency $1 \mathrm{MHz}$ at $25^{\circ} \mathrm{C}$. The dielectric constants were measured by the equation $C=\varepsilon_{0} \varepsilon_{r} \mathrm{~A} / \mathrm{d}$, where, $C$ is the capacitance, $\varepsilon_{0}$ is the permittivity of the vacuum, $\varepsilon_{\mathrm{r}}$ is the permittivity or dielectric constant of the polymer sample, $A$ is the area of the electrode plates and $d$ is the distance between top and bottom plates i.e., the thickness of the film. ${ }^{21}$ The chemical resistance test was done in various chemical environments such as aqueous $\mathrm{HCl}(10 \%)$, aqueous $\mathrm{NaOH}(5 \%)$, aqueous $\mathrm{NaCl}(15 \%)$, aqueous ethanol $(20 \%)$ and fresh water to investigate the effect of these chemicals on the cured epoxy films. The cured films were cut into small pieces and kept in $100 \mathrm{~mL}$ amber bottles containing aforesaid media at room temperature $\left(\sim 30^{\circ} \mathrm{C}\right)$. The percent of weight loss was measured after 30 days of test.

\section{Results and discussion}

\subsection{Preparation and characterization of PAD}

The PAD was prepared by Michael addition reaction between amine and acrylate as shown in scheme 1 . The excess amount of methyl acrylate in the first step and ethylene diamine in the second step was taken to ensure the complete consumption of amine and ester, respectively in the reaction. The prepared amido-

Table 1. Amount of hardener, curing time and swelling value of the thermosets.

\begin{tabular}{lccccc}
\hline & \multicolumn{2}{c}{ Amount of hardener $(\%)$} & \multicolumn{2}{c}{ Curing time at $25^{\circ} \mathrm{C}(\mathrm{h})$} & Swelling value at $25^{\circ} \mathrm{C}(\%)$ \\
\cline { 2 - 4 } Thermosets & Poly(amido-amine $)$ & PAD & Touch free & Hard cure & 72 \\
PAD0 & 50 & 0 & 8 & 9 & 27 \\
PAD5 & 20 & 5 & 2 & 6 & 15 \\
PAD10 & 20 & 10 & 1 & 5 & 18 \\
PAD15 & 20 & 15 & 0.75 & 3 & 11 \\
OPAD & 0 & 20 & 0.5 & & \\
\hline
\end{tabular}

\footnotetext{
a The swelling value of PAD0 was $16 \%$ while cured at $100^{\circ} \mathrm{C}$.
} 


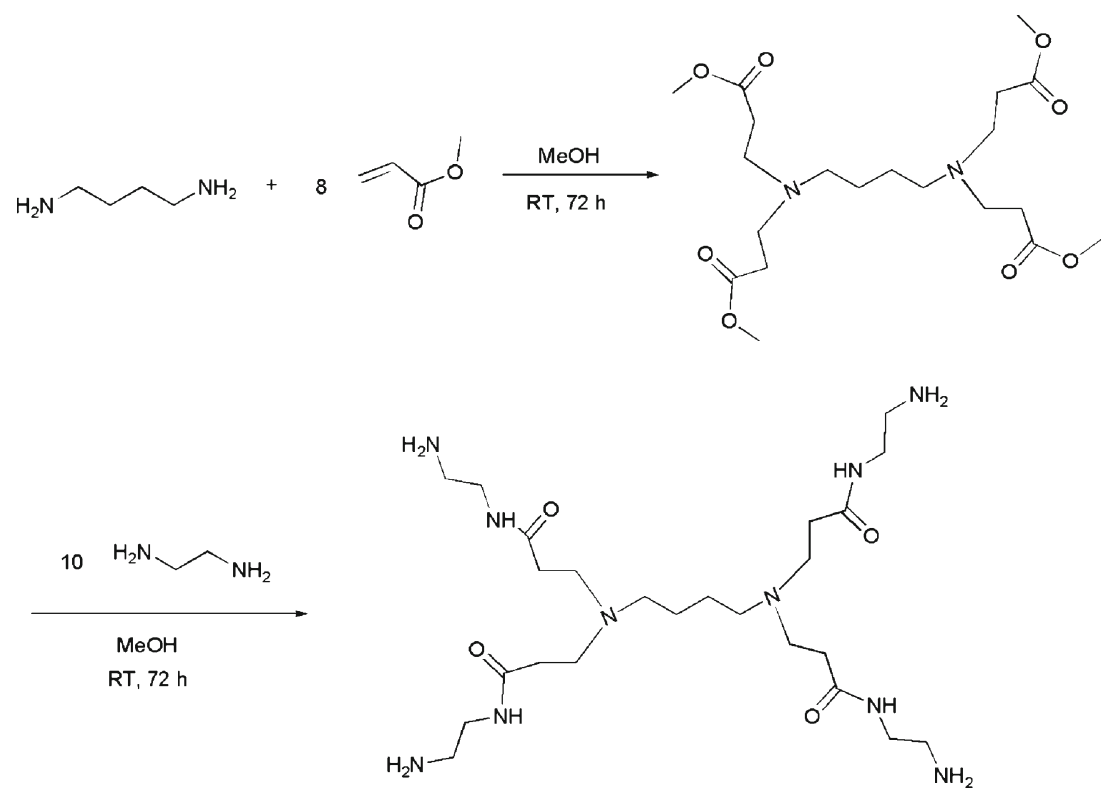

Scheme 1. Preparation of first generation poly(amido-amine) dendrimer (PAD).

amine was characterized by mainly FTIR and NMR studies. In FTIR spectrum (figure 1) the carbonyl stretching frequency of methyl acrylate at $1736 \mathrm{~cm}^{-1}$ was shifted to $1640 \mathrm{~cm}^{-1}$ which confirmed the formation of amide linkages. The other bands at 3300, 2936, 1535, 1405,1125 and $700 \mathrm{~cm}^{-1}$ were confirmed the presence of $-\mathrm{NH},-\mathrm{CH}$ stretching, $-\mathrm{NH},-\mathrm{CH}_{2}$ bending, $-\mathrm{CN}$ stretching and $-\mathrm{NH}$ out of plane stretching frequency, respectively. ${ }^{22}$ Again in the ${ }^{13} \mathrm{C}$ NMR spectrum (figure 2) the presence of chemical shift value $\delta=162 \mathrm{ppm}$ confirmed the formation amide linkages.
The peaks for two $-\mathrm{CH}_{2}$ carbons of butane diamine were observed at $\delta=50$ and $23 \mathrm{ppm}$ and for ethylene diamine at 42 and $30 \mathrm{ppm}$ (figure 2). The peaks for $-\mathrm{CH}_{2}$ carbons of methyl acrylate attached with butane diamine and carbonyl carbon were observed at $\delta=46$ and $33 \mathrm{ppm}$, respectively.

\subsection{Curing study}

Curing of the hyperbranched epoxy was done at room temperature $\left(25^{\circ} \mathrm{C}\right)$ with the combined hardener system

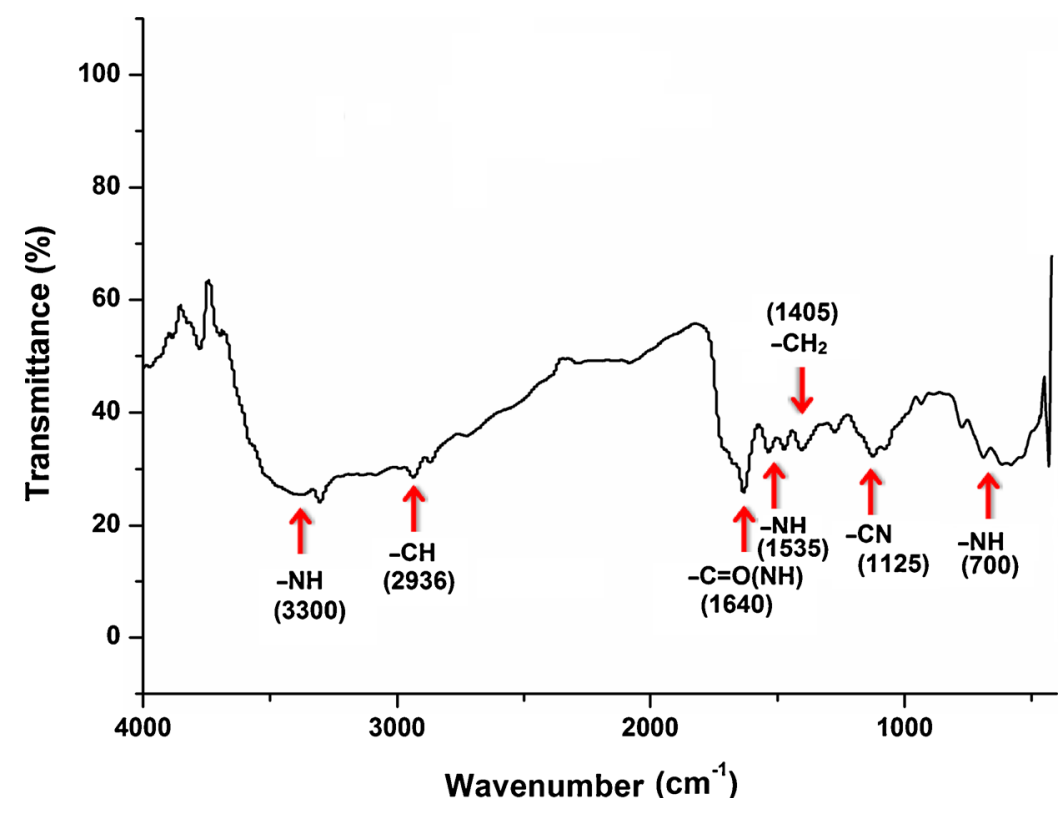

Figure 1. FTIR spectrum of PAD. 


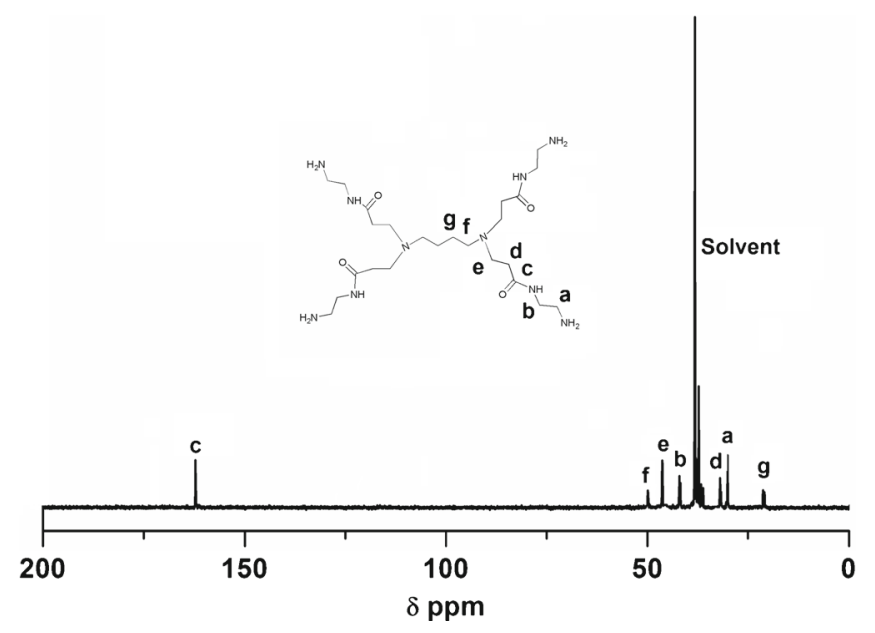

Figure 2. ${ }^{13} \mathrm{C}$ NMR spectrum of PAD.

of poly(amido-amine) and PAD. The dendritic structure with end functionalities of both the epoxy and PAD react rapidly with each other at room temperature. Thus, hyperbranched epoxy cured only with PAD resulted highly rigid and brittle thermoset (coded as OPAD). For combined hardener system PAD was taken 5, 10 and $15 \mathrm{wt} \%$ with constant $20 \mathrm{wt} \%$ linear poly(amidoamine) in each case to optimize the amount of it for the best performance of the thermoset. The decrease in curing time of the thermoset at room temperature was observed with the increase of the PAD content. The PAD contains 4 primary amine and 4 amide groups. As certain amount of amide groups of the PAD were also taken part in the curing reaction, so $10 \mathrm{wt} \%$ PAD was found to be the optimum value for curing of the hyperbranched epoxy (as shown in electronic supplementary information section). Thus, PAD10 get the highest compact crosslinked structure and showed lowest swelling value (table 1). Both the PAD and the hyperbranched epoxy have branched structures so they formed some cyclic compact structure, after curing reaction as shown in the scheme 2. PAD0 took longer curing time at room temperature and only $59 \%$ gel fraction was found after $72 \mathrm{~h}$ curing. Thus it was cured at elevated temperature like earlier study. ${ }^{19}$

\subsection{Performance of the thermosets}

3.3a Mechanical properties: The combined poly(amido-amine) cured thermosets exhibited very high tensile strength with sufficient elongation at break (table 2). Whereas solely linear poly(amidoamine) cured thermoset (PAD0) exhibited lower tensile strength with little higher elongation at break than the combined system. The dose dependent tensile strength was found on addition of PAD and the value enhanced with the increase of this hardener content. This is due to the formation of compact, rigid and cyclic thermoset of hyperbranched epoxy with PAD as shown in scheme 2. Thus, elongation at break was slightly decreased with the addition of PAD (figure 3). Toughness of the thermosets (as determined by integrating the area under stress-strain curves) remained same but it was much higher when compared to the bisphenol-A based diglycidyl ether epoxy, ${ }^{23,24}$ because of the hyperbranched architecture of the studied epoxy. The thermosets exhibited very high scratch hardness for their compact crosslinking structure, but the slight decrease in impact resistance with the addition of PAD is due to the formation of rigid cyclic crosslinking structure. The adhesive strength (lap shear tensile strength) of the thermosets increased tremendously with the addition of PAD. This is due to increase of polar functionalities (nitrogen and oxygen containing groups) along with the secondary hydroxyl groups in the system on addition of PAD. Thus, the polar-polar interactions increase with the cellulosic wood substrate whereas for metal substrate physical interlocking occurs with the hyperbranched epoxy. Thus overall the best mechanical properties were obtained for PAD10 with $10 \mathrm{wt} \%$ PAD cured thermoset because of the highest compactness of the crosslinked structure as described in the curing study. The OPAD exhibited low scratch hardness as well as low impact resistance (table 2) due to high brittleness character. Thus it was not possible to obtain the desired film for measurement of tensile strength and elongation at break for this thermoset.

3.3b Dielectric property: The variation of dielectric constant with frequency is shown in the figure 4. The dielectric constant slightly increased with the increase of PAD content because of the increment of 


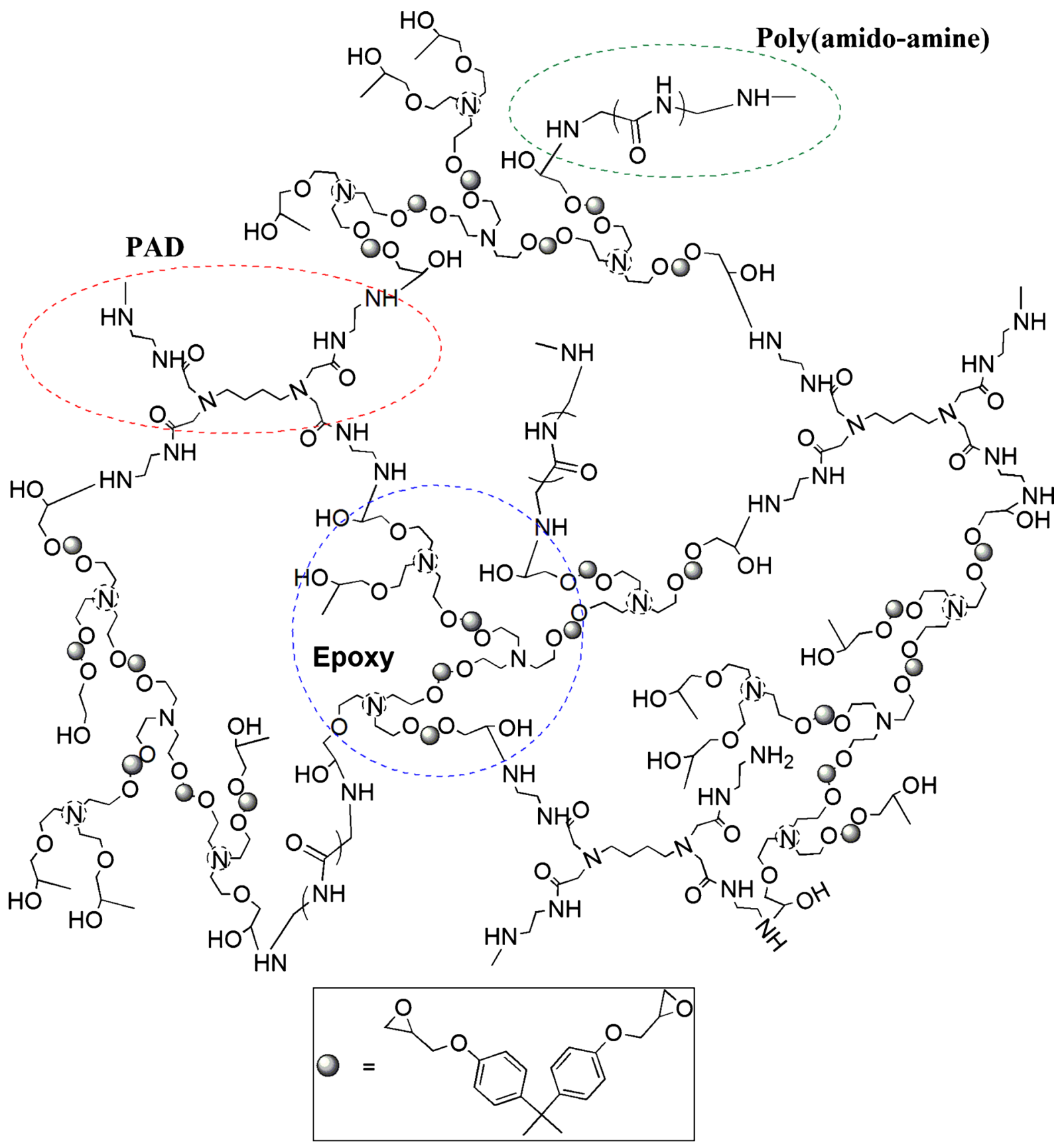

Scheme 2. Curing reaction of hyperbranched epoxy with PAD and poly(amido-amine).

total polarity of the thermoset as the PAD contain more nitrogen and oxygen containing groups in the structure. Generally, epoxy thermoset exhibits dielectric constant slightly higher compared to the other insulating materials because of the generation of secondary hydroxyl groups after curing and also hydroxyl groups in the structure. ${ }^{16}$ But here dielectric constant was low compared to the linear epoxy thermoset due to the branched unique structure of the epoxy which restricted the molar polarization at higher frequency as well as increased the free volume and thus dielectric loss was also low when compared to the bisphenol-A based diglycidyl ether epoxy. ${ }^{10,16}$ Because of the high compact crosslinked structure the moisture absorption was very low, which is advantageous for advance electrical applications.

3.3c Thermal stability: The thermal stability of the thermosets is shown in the figure 5. The thermal stability of the thermosets was remained same with the addition of PAD. This is due to the similar chemical linkages of both the hardeners. In figure 5, the PAD5 shows slightly lower thermostability because of its lower compactness of the crosslinked structure compared to other 
Table 2. Performance of the thermosets.

\begin{tabular}{|c|c|c|c|c|c|}
\hline Properties & PAD0 & PAD5 & PAD10 & PAD15 & OPAD \\
\hline Tensile strength (MPa) & 47 & 51 & 70 & 62 & - \\
\hline Elongation at break (\%) & 21 & 19 & 14 & 16 & - \\
\hline Toughness ${ }^{\text {a }}(\mathrm{MPa})$ & 413 & 396 & 438 & 400 & - \\
\hline Scratch hardness ${ }^{\mathrm{b}}(\mathrm{kg})$ & 9.0 & 9.0 & $>10.0$ & 9.5 & 5.0 \\
\hline Impact strength ${ }^{\mathrm{c}}(\mathrm{cm})$ & $>100$ & 95 & 95 & 90 & 60 \\
\hline $\mathrm{W}-\mathrm{W}$, lap-shear tensile strength ${ }^{\mathrm{d}}(\mathrm{MPa})$ & 2300 & $>3941$ & $>4226$ & $>4029$ & $>4256$ \\
\hline M-M, lap-shear tensile strength (MPa) & 2662 & 5688 & 6064 & 5964 & 7257 \\
\hline Dielectric constant at $1 \mathrm{MHz}$ (at $20^{\circ} \mathrm{C}$ ) & 3.16 & 3.22 & 3.26 & 3.32 & - \\
\hline Dielectric loss at $1 \mathrm{MHz}$ (at $20^{\circ} \mathrm{C}$ ) & 0.010 & 0.012 & 0.013 & 0.014 & - \\
\hline Moisture absorption at $25^{\circ} \mathrm{C}(\%)$ & 0.85 & 0.43 & 0.11 & 0.19 & 0.08 \\
\hline Thermal stability $\left({ }^{\circ} \mathrm{C}\right)$ & 298 & 286 & 295 & 296 & - \\
\hline
\end{tabular}

${ }^{a}$ Measured by integrating the stress-strain curves

${ }^{\mathrm{b}}$ The limit of the instrument for scratch hardness test was $10.0 \mathrm{~kg}$

${ }^{\mathrm{c}}$ The limit of the instrument for impact strength measurement was $100 \mathrm{~cm}$

${ }^{\mathrm{d}}$ In all the combine hardener cured thermosets and OPAD wood substrates were failed

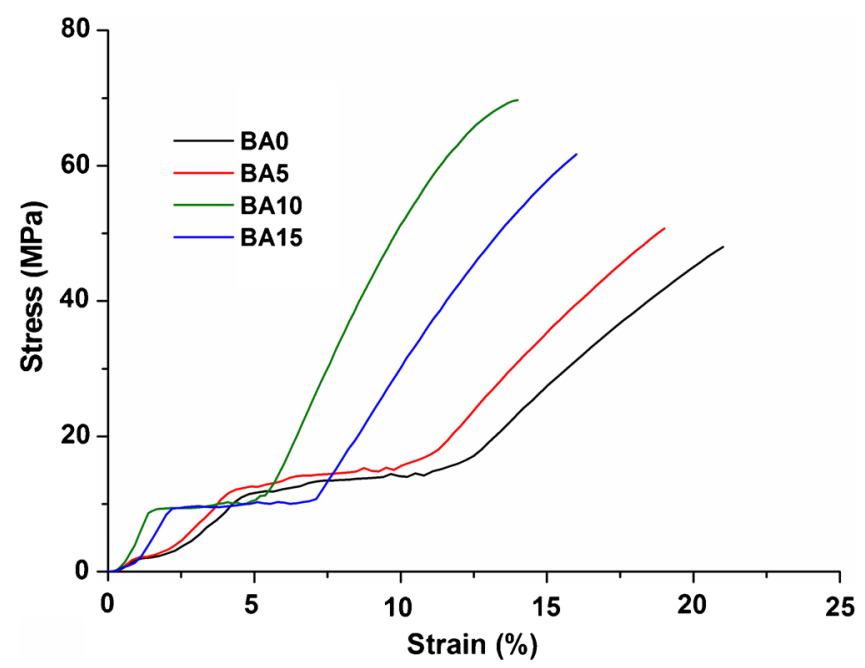

Figure 3. Stress-strain profiles of the thermosets.

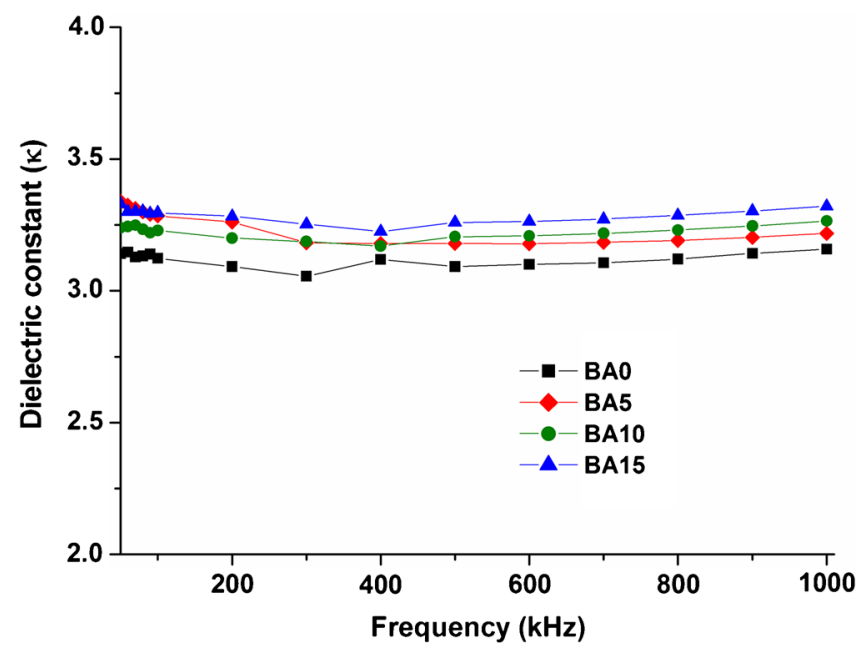

Figure 4. Dielectric constants of the thermosets with the variation of frequency. 


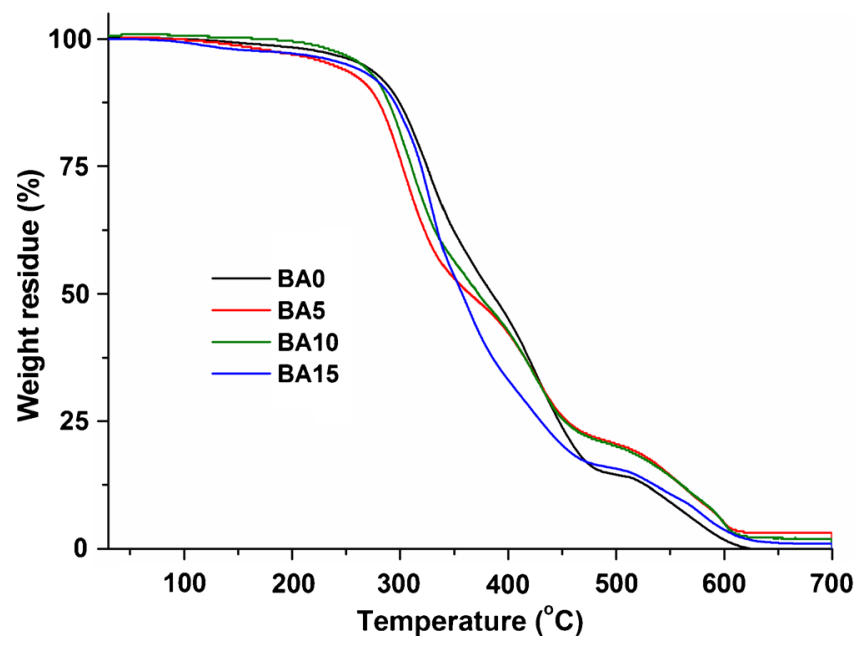

Figure 5. TGA thermograms of the thermosets.

Table 3. Weight loss (\%) of the thermosets in different chemical environments after 30 days.

\begin{tabular}{lccccc}
\hline Chemical environments & PAD0 & PAD5 & PAD10 & PAD15 & OPAD \\
\hline Aq. $\mathrm{NaOH}(5 \%)$ & 1.48 & 1.77 & 1.24 & 1.71 & 1.13 \\
Aq. HCl (10\%) & 0.90 & 1.04 & 0.50 & 0.79 & 0.38 \\
Aq. NaCl (15\%) & 0.24 & 0.34 & 0.25 & 0.27 & 0.12 \\
Aq. EtOH (20) & 0 & 0 & 0 & 0 & 0 \\
Pure water & 0 & 0 & 0 & 0 & 0 \\
\hline
\end{tabular}

studied thermosets, due to the presence of low amount of hardener. The initially degradation temperature of the thermosets $\left(\sim 300^{\circ} \mathrm{C}\right)$ is due to either degradation of aliphatic moieties or linkages whereas the second degradation $\left(\sim 450^{\circ} \mathrm{C}\right)$ is due to the aromatic moieties and the amide linkages. ${ }^{25,26}$

3.3d Chemical resistance: The chemical resistance of the thermosets in different chemical environments are shown in table 3 . They exhibited excellent chemical resistance even in strong alkali and acid media and no change in aqueous ethanol as well as in water was observed. This is only due to the high compact structure and strong chemical linkages. The highest chemical resistance was found in PAD10 among the other studied thermosets because it has the highest crosslinked and compact structure.

\section{Conclusions}

From this study, it can be concluded that the PAD is an efficient and useful curing agent at room temperature for the hyperbranched epoxy resin in combination with poly(amido-amine). The resultant thermosets exhibited high tensile strength, good thermal stability, excellent adhesive strength and low dielectric constant. Thus, this low dielectric thermoset might be potentially useful for electronic devices operated under high frequency, microelectronic devices, electrical packaging, etc.

\section{Supplementary information}

Calculation for the equivalent amount of hardener and the general structure of the hyperbranched epoxy are available as supplementary information (figures S1 S5 and schemes S1 - S2) at www.ias.ac.in/chemsci website.

\section{Acknowledgements}

The authors thank the Naval Research Board (NRB) for financial assistance through the grant No. DNRD/05/4003/NRB/251 dated 29.02.12, SAP University Grants Commission (UGC), India through grant No. F.3-30/2009(SAP-II) and FIST program-2009 Department of Science and Technology (DST), India through the grant No. SR/FST/CSI-203/209/1 dated 06.05.2010. 


\section{References}

1. Lee H and Neville K 1967 Handbook of epoxy resins; (New York: McGraw-Hill)

2. Karak N 2009 Fundamentals of polymers: Raw materials to applications; (New Delhi: PHI Learning Pvt. Ltd.)

3. Wu D, Wu L, Wang J, Sun Y and Zhang M 2011 Mater. Chem. Phys. 128274

4. Wan J, Li Z, Bu Z Y, Fan H and Li B G 2012 Mater. Chem. Phys. doi:10.1016/j.matchemphys.2012.11.060

5. Tomalia D A and Dewald J R 1986 US Patent: 4,568,737

6. Yiyun C, Dazhu C, Rongqiang F and Pingsheng H 2005 Polym. Int. 54495

7. Young H K 1998 J. Polym. Sci. Part A: Polym. Chem. 36 1685

8. Voit B 2000 J. Polym. Sci. Part A: Polym. Chem. 382505

9. Jikei M and Kakimoto M 2001 Prog. Polym. Sci. 26 1233

10. Voit B 2005 J. Polym. Sci. Part A: Polym. Chem. 43 2679

11. Gao C and Yan D 2004 Prog. Polym. Sci. 29183

12. Fu J F, Shi L Y, Yuan S, Zhong Q D, Zhang D S, Chen Y and Wu J 2008 Polym. Adv. Technol. 191597

13. Zhang D and Jia D 2006 J. Appl. Polym. Sci. 1012504
14. Emrick T, Chang H T and Frechet J M J 1999 Macromolecules 326380

15. Emrick T, Chang H T and Frechet J M J 2000 J. Polym. Sci. Part A: Polym. Chem. 384850

16. Nalwa H S 1999 Handbook of low and high dielectric constant materials and their applications, 1, (Boston: Academic Press)

17. Lin C H, Jiang Z R and Wang C S 2002 J. Polym. Sci. Part A: Polym. Chem. 404084

18. Pitt C F, Barth B P and Godard B E 1957 IRE Transactions on Component Parts 4110

19. De B and Karak N 2013 J. Mater. Chem. A 1348

20. Das G and Karak N 2009 Prog. Org. Coat. 6659

21. Thakur S and Karak N 2013 Prog. Org. Coat. 76157

22. Pavia D L, Lampman G M, Kriz G S and Vyvyan J R 2010 Spectroscopy, (New Delhi: Cengage Learning India Pvt. Ltd.)

23. Jyotishkumar P, Pionteck J, Hassler R, George S M, Cvelbar U and Thomas S 2011 Ind. Eng. Chem. Res. 50 4432

24. Ratna D, Samui A B and Chakraborty B C 2004 Polym. Int. 531882

25. Roy B and Karak N 2012 J. Mater. Res. 271806

26. van Bennekom A C M, Willemsen P A A T and Gaymanst R J 1996 Polymer 375447 